\title{
Discouraging transplant results
}

On the back of promising animal studies, several trials of neural transplantation for the treatment of neurodegenerative diseases have been initiated over the past decade. Last year the first long-term evaluations of fetal dopaminergic neuron transplantation in patients with Parkinson's disease produced mixed results: at least some of the grafted cells seemed to have acquired signs of disease pathology (see Further reading). Now, Cicchetti et al. report that similar neural transplants in patients with Huntington's disease (HD)

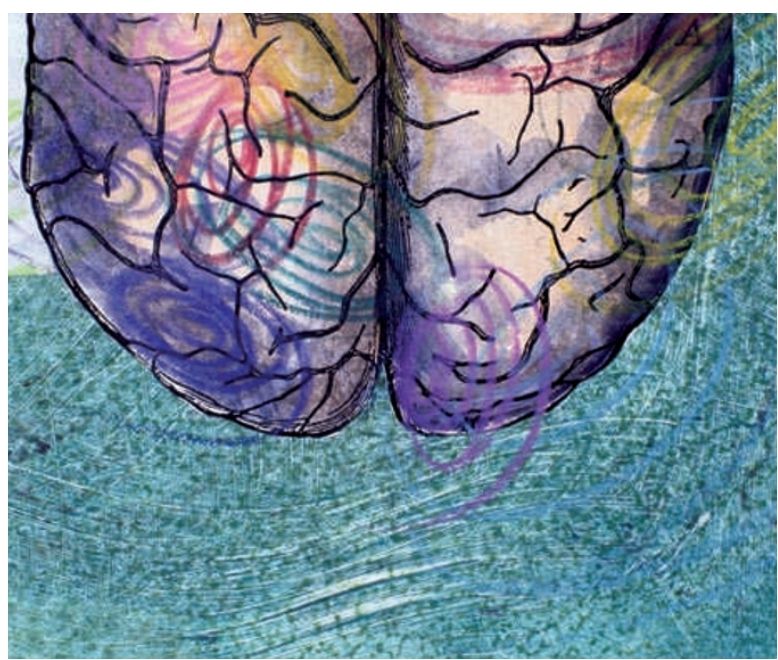

also showed signs of disease-like deterioration.

The authors evaluated the postmortem brain tissue of three patients with HD who received multiple grafts containing embryonic striatal tissue in the caudate and putamen, the principal brain regions affected in HD, 10 years before their death. Histology revealed that, although the caudate grafts died, several putamen grafts survived in two patients. Nevertheless, brain atrophy in the putamen of these patients was comparable to that in age-matched control patients.

Immunohistochemistry showed that the surviving grafts expressed markers of striatal interneurons and projection neurons and that host neurons had made synaptic contacts with the grafted neurons. However, there were morphological and immunohistochemical signs of neuronal degeneration in the grafts, which were mainly confined to the medium spiny projection neuron population, the main source of striatal neuronal output. Possible contributing factors to the graft degeneration include microglial activation, excitotoxicity and a poor trophic environment. Indeed, the authors also observed markers of an inflammatory response, including microglia infiltration, within the graft.

Interestingly, the grafts were free of aggregates of the disease-related proteins ubiquitin and huntingtin, suggesting that the graft degeneration is due to factors in the host environment rather than a direct effect of these proteins.

These results are discouraging for both researchers and patients, who had hoped that cell transplantation would provide an effective treatment for this currently incurable disease. However, a possible 'silver lining' is that the results may provide important information on potential disease mechanisms - such as microglial activation and/or excitotoxicity which might contribute to more promising therapeutic approaches in the future.

Katherine Whalley

ORIGINAL RESEARCH PAPER Cicchetti, F. et al. Neural transplants in patients with Huntington's disease undergo disease-like neuronal degeneration. Proc. Natl Acad. Sci. USA 106, 12483-12488 (2009)

FURTHER READING Brundin, P. et al. Research in motion: the enigma of Parkinson's disease pathology spread. Nature Rev. Neurosci. 9, 741-745 (2008) 